\title{
Reduced Path Successive Cancellation List Decoding for Polar Codes
}

\author{
Walled Khalid Abdulwahab*, Abdulkareem Abdulrahman Kadhim \\ College of Information Engineering, Al-Nahrain University, Baghdad, Iraq \\ Received 05 September 2020; received in revised form 19 October 2020; accepted 18 December 2020
}

DOI: https://doi.org/10.46604/ijeti.2021.6376

\begin{abstract}
Polar codes have already been adopted in 5G systems to improve error performance. Successive cancellation list (SCL) decoding is usually used at the decoder and involves lengthy processing. Therefore, different methods have been developed to reduce an SCL decoder's complexity. In this paper, a reduced path successive cancellation list (RP-SCL) decoder is presented to reduce this complexity, where some decoding paths are pruned. The pruning is achieved by using three different thresholds: two for the path metric and one for the pruning depth in the decoding tree. An optimization procedure is considered to determine the optimum settings for these thresholds. The simulation tests are carried out over models of an additive white Gaussian noise channel and a fading channel by using $5 \mathrm{G}$ environments. The results reveal that the proposed RP-SCL decoder provides the complexity reduction in terms of the average number of processed paths at high SNR. Additionally, the computational complexity and the memory requirements decrease.
\end{abstract}

Keywords: polar codes, SCL, tree pruning, 5G, mmWave

\section{Introduction}

Polar codes, introduced by Erdal Arikan in 2009, are linear block codes that utilize channel polarization to achieve the channel capacity of symmetric binary-input discrete memoryless channels (B-DMC) [1]. Polar codes with successive cancellation (SC) decoders have achieved the attractive performance for long coded block length (N) with a complexity of $\mathrm{O}\left(\mathrm{N} \cdot \log _{2} \mathrm{~N}\right)[2]$.

SC utilizes a binary tree for representing polar codes in the decoding process. It navigates the tree by picking the left edges until the leaf nodes are reached. If the node is a frozen one, then it is set to zero. In the case of information nodes, threshold detection is used to estimate the bit value, and SC backtracks to the right edge [3]. When a node gets the estimation values from both of its children, it combines the estimated values and pushes its own estimation to its parent node. Thus, SC navigates the whole decoding tree until all of the leaf nodes are decoded.

Unfortunately, the performance of an SC decoder is not as good as that of maximum likelihood (ML) decoding with short and moderate coding lengths, which is very important for 5G applications [4-5]. To overcome this weakness, successive cancellation list (SCL) decoding was proposed to improve the performance of polar codes for low and moderate $\mathrm{N}[6]$.

In the SCL approach, a set of SC decoders works together and maintains different candidate paths. At the leaf nodes and in the case of information nodes, both bit values 0 and 1 are considered, and the number of decoding paths are duplicated. This process continues, and the number of decoding paths is doubled at each information node [6]. A pruning process is used to limit the number of paths to $\mathrm{L}$ paths, where $\mathrm{L}$ is the decoding list size.

\footnotetext{
* Corresponding author. E-mail address: walled.khalid@ coie-nahrain.edu.iq

Tel.: +964-770-1323837
} 
Polar codes with an SCL decoder compete in performance with other modern channel coding schemes [7-8]. The improvement in performance achieved by SCL is at the cost of increasing the decoding complexity from $\mathrm{O}\left(N . \log _{2} N\right)$ to $\mathrm{O}\left(L \cdot N \cdot \log _{2} N\right)$. This represents a critical issue for most modern communication systems. Many studies have focused on reducing the SCL decoder complexity, which is substantial for 5G devices [4-5]. These works used different approaches to achieve complexity reduction.

The decoding tree path pruning approach was proposed in [9-10] to reduce the number of active decoding paths. At each decoding level, a threshold condition is determined, and any path with a metric which does not satisfy the threshold will be removed. The threshold is specified on the basis of the path metric values and a certain parameter. This parameter is determined at each decoding level depending on a list including pruned paths, which have a significant impact on the performance of the decoder. The list must be updated at each level. This list and its updating mechanism can add overhead to the decoding process.

Decoding tree partitioning represents another approach for reducing SCL decoder complexity. In [11-13], a partitioning algorithm to break down the decoding tree for SCL was proposed and developed to reduce the required memory space. In [14-16], the approaches for reducing the SCL computational complexity and latency were presented by removing the redundant and unnecessary calculations or by using the specific sub-codes and nodes in the polar code.

Most of the above-mentioned works are either concerned with reducing the memory requirement for the decoder at the cost of increasing the decoding latency, as in [11-13], or interested in reducing the computational complexity of the decoder, as in [14-16]. The decoding tree pruning approach proposed in [9-10] reduced the total number of processed paths, which in turn reduced the computational complexity, required memory, and the decoding latency. The limitation of the later works was the added overhead to the decoding process by the updating mechanism for the pruning threshold value; in addition, the proposed system was evaluated over an additive white Gaussian noise (AWGN) channel only. In this work, the idea of reducing the complexity of an SCL decoder by tree pruning was utilized. The purpose is to reduce the average number of processed paths and in turn reduces the SCL decoder complexity with the minimum added overhead. Three thresholds were considered to control the pruning of the decoding paths. These thresholds were determined on the basis of three different parameters.

The values of these parameters were optimized on the basis of a fixed value of the signal-to-noise ratio (design SNR) in order to achieve the maximum possible reduction in the decoding complexity accompanied by the minimum degradation in the BER performance. This optimization process was carried out before the actual data transmission; thus, no added overhead was required as in the previous works. The proposed decoder, which is called reduced path SCL or RP-SCL, was evaluated over an AWGN channel and a fading channel with a 5G environment. RP-SCL succeeds in achieving considerable reduction in the decoding complexity as compared to that of the standard SCL decoder.

The rest of this paper is organized as follows: In Section 2, the proposed RP-SCL decoder is described along with its complexity reduction. The considered 5G environment and channel model are presented in Section 3 . The results of the proposed RP-SCL decoder over the considered channel models are presented and discussed in Section 4 . Finally, the conclusions of the work are given in Section 5.

\section{Proposed Reduced Path SCL Decoder}

The SC and SCL decoding processes are identical in their initial steps but differ in the mechanisms of decoding the data bits. Instead of having the final decoding decision for the current data bit based on the thresholding decision used in SC, two paths are created with the two possible values for the decoded bit ('0' and ' 1 ') in the SCL decoder. Clearly, the number of paths is duplicated at each decoding step. 
Consider a decoding tree for an SCL decoder with $\mathrm{N}$ levels and a maximum of $L$ paths at a time. A pruning process removing the worst paths is used to limit the number of decoding paths to just $L$ paths on the basis of the path metric (PM) defined in [17], which can be obtained by:

$$
C_{l}^{(i)} \triangleq \sum_{j=0}^{i} \ln \left(1+e^{-\left(1-2 \hat{u}_{j}[l]\right) \cdot L_{n}^{(j)}[l]}\right)
$$

where $C_{l}^{(i)}$ is the PM of the $l^{\text {th }}$ path at the $i^{\text {th }}$ level, $\hat{u}_{j}[l]$ is the decoded data bit at the $j^{\text {th }}$ level $(0 \leq j \leq i)$ and $l^{\text {th }}$ path, $n$ is the power of the Kronecker matrix used to generate the codeword, and $L_{n}^{(i)}[l]$ is the $\log \operatorname{likelihood}$ ratio (LLR) for bit $u_{i}$ defined by the following equation [17]:

$$
L_{l}^{(i)}=\ln \left(\frac{w_{n}^{(i)}\left(y, \hat{u}^{i-1}[l] \mid 0\right)}{w_{n}^{(i)}\left(y, \hat{u}^{i-1}[l] \mid 1\right)}\right)
$$

where $\hat{u}^{i-1}$ is the past route of the path and $w_{n}^{(i)}$, with $i \in N$ and $\mathrm{N}=2^{n}$, is a binary discreet memoryless channel with $u$ and $y$ as its input and output, respectively. Investigating the SCL decoding process, one may conclude that most of the correct paths are those with the least PM lying at the beginning of the list.

Table 1 illustrates the distribution of the correct paths over the SCL decoding list index for 782 transmitted codewords by using a polar code with a coded block length $(N)$ of 256 bits and a data block length $(K)$ of 128 bits (i.e. PC $(256,128))$ and with a list size of 8 for two different SNR values of 1 and $3 \mathrm{~dB}$ over an AWGN channel. This table clarifies that a majority of the correct paths are those associated with the low indices. The case of 3-dB SNR shows that almost all the correct paths have an index of 1 .

Table 1 Number of correct paths at each list index for $L=8$ over AWGN channel

\begin{tabular}{|c|c|c|c|c|c|c|c|c|}
\hline Index & 1 & 2 & 3 & 4 & 5 & 6 & 7 & 8 \\
\hline SNR $=1 \mathrm{~dB}$ & 669 & 59 & 23 & 14 & 7 & 5 & 3 & 2 \\
\hline SNR $=3 \mathrm{~dB}$ & 774 & 8 & 0 & 0 & 0 & 0 & 0 & 0 \\
\hline
\end{tabular}

According to Table 1, the possibility of having redundant paths is high. It allows for the further pruning or discarding of a number of paths usually with a high PM without, however, affecting the performance as shown later. Thus, a reduced path SCL (RP-SCL) decoder that utilizes three thresholds is proposed in this work. These thresholds are derived on the basis of three different parameters. The optimum parameter settings are considered after a thorough investigation. The path pruning in RP-SCL is established after the SCL conventional pruning is performed. In the remaining parts of this section, the threshold values are clarified along with their parameter settings.

\subsection{Reduction of terminated branches using maximum metric}

In conventional SCL, the $L$ paths are arranged according to their path metrics with the maximum path metric $\left(C_{m a x}\right)$ corresponding to the worst path. Let $\left\{C_{l}\right\}$ be the set of path metrics of all the $\mathrm{L}$ paths at the given decoding level. The proposed RP-SCL decoding algorithm reduces the number of processing paths by using three different thresholds. The first threshold $\left(T_{\alpha}\right)$ is determined according to $C_{\max }$ which can be obtained by:

$$
T_{\alpha}=C_{\max }-\alpha \cdot C_{\max }, \quad 0<\alpha<1
$$

In the RP-SCL decoding algorithm, any path with $C_{l}$ which is greater than $T_{\alpha}$ is discarded. Thus, the number of discarded paths at each level is not fixed and usually depends on the value of $T_{\alpha}$ and the path metrics. Clearly, when the average number of processed paths $\left(L_{a v}\right)$ by RP-SCL is less than $L$, RP-SCL has an advantage over the conventional SCL decoder. 
To find the optimum value of the threshold level $T_{\alpha}$ for the given code, $\alpha$ is varied from 0 to 1 , and the corresponding $L_{a v}$ is determined. The optimal value of $\alpha\left(\alpha_{o p t}\right)$ corresponds to the least $L_{a v}$. As $\alpha$ increases, $L_{a v}$ decreases until a turning point is reached at $\alpha_{\text {opt }}$. After this point, $L_{a v}$ then starts to increase as $\alpha$ approaches its maximum value of unity.

The decrease in $T_{\alpha}$ is due to the high value of $\alpha$ results in small $L_{a v}$. A problem may occur when all the metrics are greater than $T_{\alpha}$ so that all the paths must be pruned, which leads to a failure of the decoding process. In such a case, all the $L$ paths are considered in the decoding process. To overcome this limitation, a new path reduction mechanism is considered, as described later (Subsection 2.3).

\subsection{Reduction of decoding depth}

A reduction in $L_{a v}$ usually leads to a performance degradation of the RP-SCL decoding. To reduce this degradation, the depth of the decoding tree, at which the pruning is started, is controlled by $D_{\beta}$ where:

$$
D_{\beta}=\lceil\beta . K\rceil, \quad 0<\beta<1
$$

where $K$ is the length of the data block at the input of the polar encoder, and $\lceil\beta . K\rceil$ is the ceiling of $\beta . K$.

When the decoding depth reaches $D_{\beta}$ in terms of the number of data bits, the RP-SCL pruning is executed. When the value of $\beta$ is close to 0 , the pruning process starts at the top of the decoding tree. Thus, a further reduction in complexity is achieved. As the value of $\beta$ increases, the pruning starts at the relatively low levels of the decoding tree. When the $\beta$ value is close to unity, $L_{a v}$ is increased, and the decoder performance is improved. A compromise between $L_{a v}$ and the decoding performance can be achieved to determine $\beta_{\text {opt }}$.

\subsection{Reduction of terminated branches using metrics' mean}

To overcome the problem mentioned in the subsection 2.1 regarding the pruning of all the paths by a small value of $T_{\alpha}$, a third threshold level test based on the mean value of the path metrics $\bar{C}_{l}$ is used to reduce the decoding complexity while maintaining the acceptable performance. The new threshold, which is called $T_{\gamma}$, is used only for the case when the $T_{\alpha}$ threshold tests the pruning of all the paths at a given level. The threshold test of $T_{\gamma}$ is similar to that of $T_{\alpha}$, where any path with $C_{l}$ is greater than $T_{\gamma}$, is pruned. The threshold level $T_{\gamma}$ is defined as:

$$
T_{\gamma}=\gamma \cdot \bar{C}_{l}, \quad \gamma \geq 1
$$

Furthermore, the mean value of the metric $\bar{C}_{l}$ is calculated as:

$$
\bar{C}_{l}=\frac{\sum_{j=1}^{L} C_{j}^{(i)}}{L}
$$

where $i$ is the decoding level. The effect of $T_{\gamma}$ on the RP-SCL pruning process is controlled by the parameter $\gamma$. As $\gamma$ increases, $L_{a v}$ increases, and the decoder performance improves. In contrast, when $\gamma$ decreases, $L_{a v}$ decreases at the cost of performance degradation. Because $\alpha_{o p t}$ usually achieves a good reduction in $L_{a v}$ at the cost of noticeable performance degradation, the range of $\gamma$ is considered to be $\geq 1$ in order to mitigate the effect of $\alpha_{\text {opt }}$ on the decoder performance. The optimum setting of $\gamma$ depends on both the complexity $\left(L_{a v}\right)$ and the bit error rate (BER) performance of the decoder.

\subsection{Full operation of the proposed RP-SCL decoder}

The operation of the RP-SCL decoder relies on determining the optimum values for the three parameters $(\alpha, \beta$, and $\gamma)$ at the assumed SNR (called design SNR) prior to any transmission, as shown in algorithm 1. When the actual transmission 
begins, RP-SCL uses the optimum parameters to determine the threshold tests in order to use them in the decoding process, as described previously.

The optimum values of $\alpha, \beta$, and $\gamma$ are determined by varying their values according to the given ranges and then selecting the value that produces the least $L_{a v}$ as follows: First, $\alpha_{o p t}$ is determined, while $\beta$ and $\gamma$ are set to 0 and 1 , respectively. Then, $\alpha_{\text {opt }}$ along with $\gamma=1$ is used to determine $\beta_{\text {opt }}$. Finally, the value of $\gamma_{\text {opt }}$ is determined, while $\alpha$ and $\beta$ are set to their optimum values $\alpha_{o p t}$ and $\beta_{\text {opt }}$, respectively. A simplified block diagram of the data transmission system with the related components of the polar coding and decoding is shown in Fig. 1.

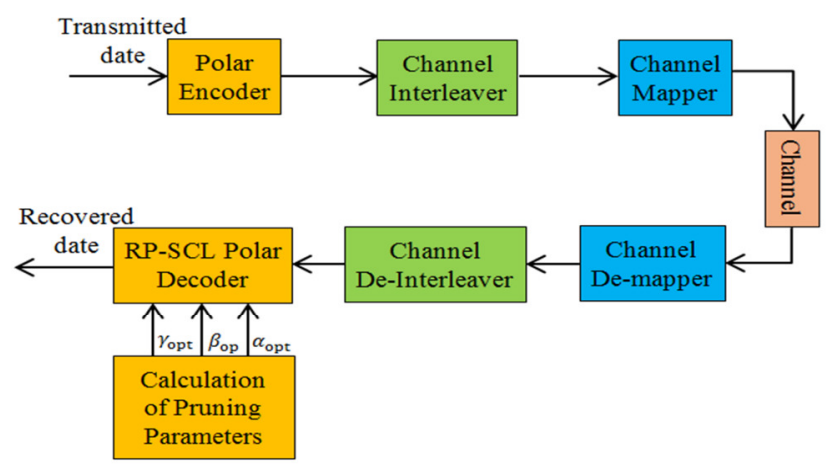

Fig. 1. The simplified block diagram of the data transmission system

Algorithm 1 The determination of the optimum pruning parameters and the operation of RP-SCL

// Parameter setting phase, determining the optimum values for the pruning parameters on the basis of specific design SNR

// Steps for determining $\alpha_{o p t}$

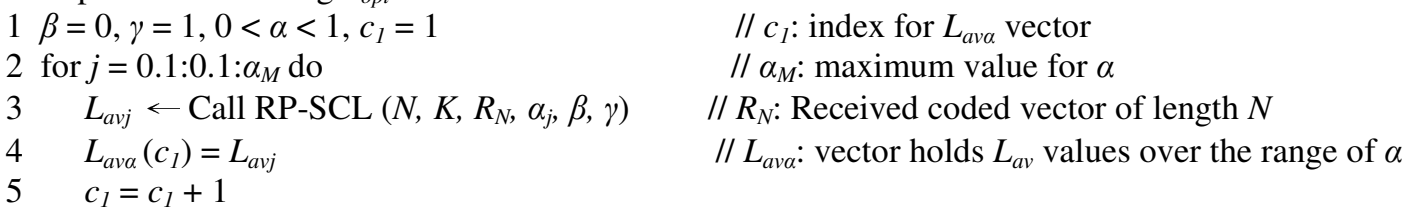

6 End for

$7 \alpha_{\text {opt }} \leftarrow \min \left(L_{a v a}\right)$

// Steps for determining $\beta_{\mathrm{opt}}$

$8 \alpha=\alpha_{\text {opt }}, \gamma=1,0 \leq \beta<1, c_{2}=1 \quad / / c_{2}$ : index for $L_{a v \beta}$ and $B E R_{\beta}$ vector

9 for $j=0: 0.1: \beta_{M}$ do

$/ / \beta_{M}$ : maximum value for $\beta$

$10 \quad\left[d_{r}, L_{a v j}\right] \leftarrow \operatorname{Call} \mathrm{RP}-\operatorname{SCL}\left(N, K, R_{N}, \alpha_{o p t}, \beta_{j}, \gamma\right) \quad / / d_{r}$ : received data

$11 \operatorname{BER}_{\beta}\left(c_{2}\right)=$ compare $\left(d_{s}, d_{r}\right)$

$/ / d_{s}$ : sent data, $B E R_{\beta}$ : vector holds BER values over the range of

$\beta$

$12 L_{a v \beta}\left(c_{2}\right)=L_{a v j}$

$/ / L_{a v \beta}$ : vector holds $L_{a v}$ values over the range of $\beta$

$13 \quad c_{2}=c_{2}+1$

14 End for

$15 \beta_{\text {opt }} \leftarrow$ Compromise $\left(L_{a v \beta}, B E R_{\beta}\right)$

// Steps for determining $\gamma_{\text {opt }}$

$16 \alpha=\alpha_{o p t}, \beta=\beta_{\mathrm{opt}}, \gamma \geq 1, c_{3}=1$

17 for $\gamma_{j}=1: 0.025: \gamma_{M}$ do

$18\left[d_{r}, L_{a v j}\right] \leftarrow$ Call RP-SCL $\left(N, K, R_{N}, \alpha_{o p t}, \beta_{o p t}, \gamma_{j}\right)$

$19 \operatorname{BER}_{\gamma}\left(c_{3}\right)=$ compare $\left(d_{s}, d_{r}\right)$

$20 \quad L_{a v \gamma}\left(\mathrm{c}_{3}\right)=L_{a v j}$

$21 \quad c_{3}=c_{3}+1$

23 End for

$24 \gamma_{\text {opt }} \leftarrow$ Compromise $\left(L_{a v \gamma}, B E R_{\gamma}\right)$

// Actual transmission phase, SCL decoding process

$25\left[d_{r}, L_{a v}\right] \leftarrow \operatorname{RP}-\operatorname{SCL}\left(N, K, R_{N}, \alpha_{\text {opt }}, \beta_{\text {opt }}, \gamma_{\text {opt }}\right)$

// Determine $\beta_{\text {opt }}$ on the basis of compromise between $L_{a v \beta}$ and $B E R_{\beta}$

$/ / c_{3}$ : index for $L_{a v \gamma}$ and $B E R_{\gamma}$ vector

$/ / \gamma_{M}$ : maximum value for $\gamma$

// $B E R_{\gamma}$ : vector holds BER values over the range of $\gamma$

$/ / L_{a v \gamma}$ : vector holds $L_{a v}$ values over the range of $\gamma$

// Determine $\gamma_{\text {opt }}$ on the basis of compromise between $L_{a v \gamma}$ and $B E R_{\gamma}$

$/ / L_{a v}$ : average number of processed paths with RP-SCL decoding

\begin{tabular}{llc}
\hline & & Subroutine $1 \mathrm{RP}-\operatorname{SCL}\left(N, K, R_{N}, \alpha_{\text {opt }}, \beta_{\mathrm{opt}}, \gamma_{\mathrm{opt}}\right)$ \\
\hline 1 & $c_{4}=1$ & $/ / c_{4}:$ vector index \\
2 & For $i=1,2, \ldots, N$, do & $/ / N$ : coding length \\
3 & If $i \in F$ then & $/ / F$ : Frozen bit set
\end{tabular}




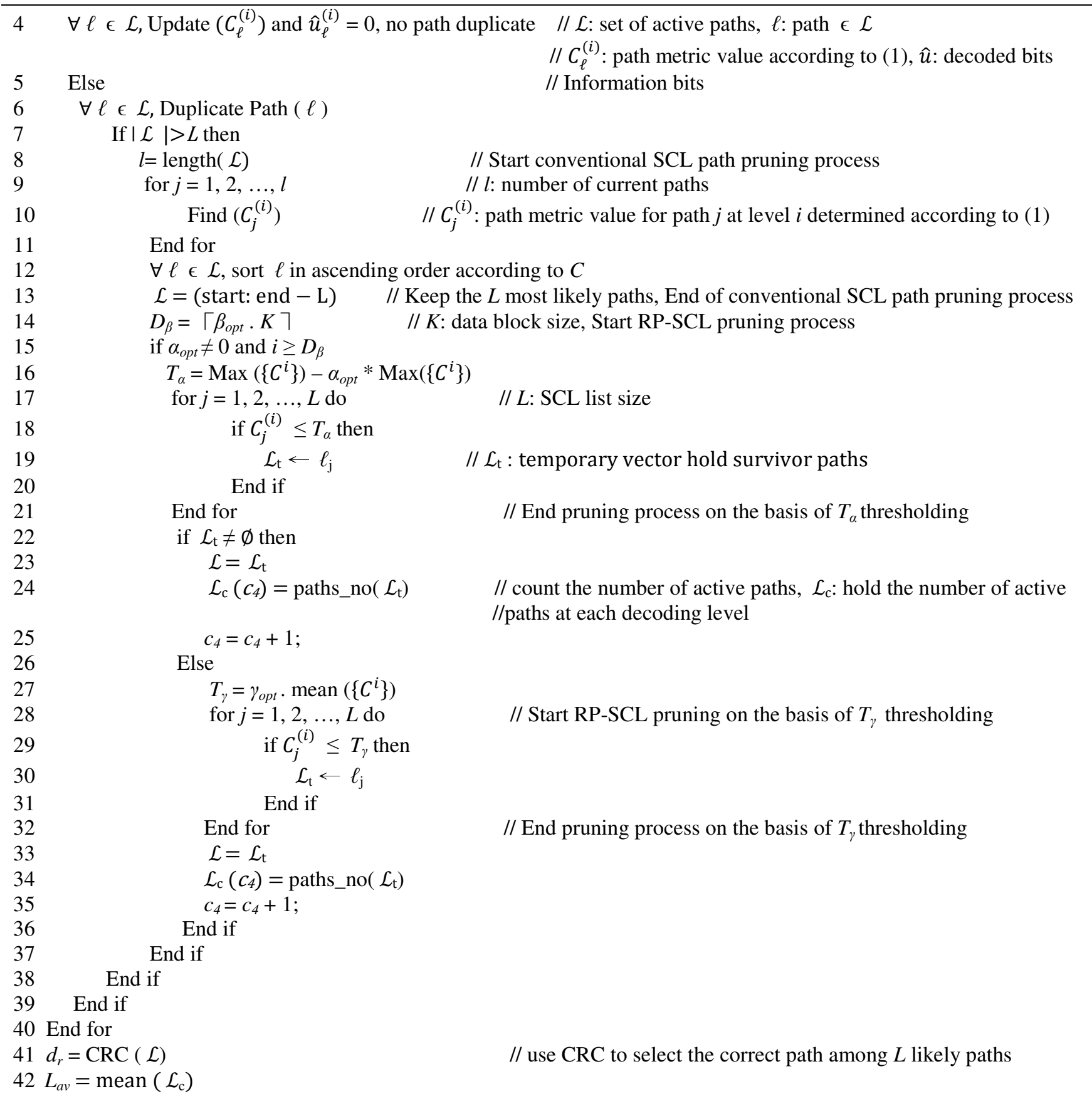

\section{5G Environments and Channel Model}

5G system environments and channel models were used in the work to evaluate the BER performance of the proposed RP-SCL decoder. The main features of the 5G transmission system were the use of the following: millimetre wave (mmWave) frequency band, orthogonal frequency division multiplexing (OFDM), multilevel phase shift keying (MPSK) or multilevel quadrature amplitude modulation (MQAM), and multi-input multi-output (MIMO) technology [18-22].

The related parameters for the mentioned techniques actually used in the simulation are presented in Table 2 . The related components of these techniques are presented within the signal mapping and de-mapping blocks of Fig. 1. The justification for the used parameter values and details of their settings could be found elsewhere [20, 23].

The channel model considered here was approved by $3 \mathrm{GPP}$ and defined over the frequency range of $0.5 \mathrm{GHz}$ to 100 $\mathrm{GHz}$ for the maximum bandwidth of $2 \mathrm{GHz}$ [24]. Model D (indoor office with a short delay profile) was considered using the tapped delay line channel model. The simulation parameters of the fading channel model are given in Table 3. 
Table 2 The simulation parameters of $5 \mathrm{G}$ transmission system

\begin{tabular}{|c|c|}
\hline Parameter & Value or Type \\
\hline Carrier Frequency & $28 \mathrm{GHz}$ \\
\hline Bandwidth & $50 \mathrm{MHz}$ \\
\hline Modulation & QPSK \\
\hline No. of Subcarriers in OFDM & 512 \\
\hline Length of Cyclic Prefix & 36 \\
\hline Sub-Carrier Spacing & $120 \mathrm{kHz}$ \\
\hline OFDM Frame Duration & $8.33 \mu \mathrm{s}$ \\
\hline MIMO Type & $4 \times 4(\mathrm{STBC})$ \\
\hline
\end{tabular}

Table 3 The simulation parameters of fading channel model

\begin{tabular}{|c|c|}
\hline Parameter & Value \\
\hline No. of Multipaths & 3 \\
\hline Path Gains & {$\left[\begin{array}{lll}-0.2 & -18.8 & -21\end{array}\right] \mathrm{dB}$} \\
\hline Normalized Path Delay & {$\left[\begin{array}{llll}0 & 0.035 & 0.612\end{array}\right]$} \\
\hline Delay Spread & $16 \mathrm{~ns}$ \\
\hline K-Factor & $13.3 \mathrm{~dB}$ \\
\hline Velocity & $3 \mathrm{~km} / \mathrm{h}$ \\
\hline Max. Doppler Shift & $77.784 \mathrm{~Hz}$ \\
\hline
\end{tabular}

\section{Performance Evaluation of RP-SCL Decoder}

The proposed RP-SCL decoder was used for polar code PC $(256,128)$ with two list sizes of 8 and 32 . The selected block length for the polar code was among the interesting lengths in two $5 \mathrm{G}$ use cases: the massive machine type communication (mMTC) and the ultra-reliable low latency (URLL) [25]. RP-SCL with the considered polar code was simulated by using QPSK and OFDM modulation with the related transmission parameters, as shown in Table 2.

Two channel models are used: the additive white Gaussian noise (AWGN) channel and a fading channel model in the mmWave frequency band-related specifications, as shown in Table 3. The proposed RP-SCL decoder together with the conventional SCL decoder is simulated by using Matlab R2019a version 9.6. The results of the simulation are presented in this section followed by the discussions related to the computational complexity and the memory requirements of RP-SCL.

\subsection{Simulation tests and results}

The extensive simulation tests are conducted in this work which covers the estimation of the optimum pruning parameters for each channel and the performance evaluation of the proposed RP-SCL decoder together with that of the standard SCL decoder for the sake of comparison. For the AWGN channel, an SNR of $3.1 \mathrm{~dB}$ is considered the design SNR to determine the optimum pruning parameters, where the SCL decoder for the adopted polar code achieves a BER of $10^{-4}$. This BER value is considered to be acceptable here. The corresponding SNR for the fading channel is approximately 24.25 $\mathrm{dB}$.

The estimation of the optimum pruning parameters started by finding $\alpha_{\text {opt }}$ first as mentioned before. This is followed by determining $\beta_{\text {opt }}$ and finally $\gamma_{\text {opt }}$ over each channel. Fig. 2 illustrates the variation of $L_{a v}$ against $\alpha$ with $L=8$. The value of $\alpha$ that yields the minimum $L_{a v}\left(\alpha_{o p t}\right)$ is approximately 0.6 with an average number of processed paths of 4.2.

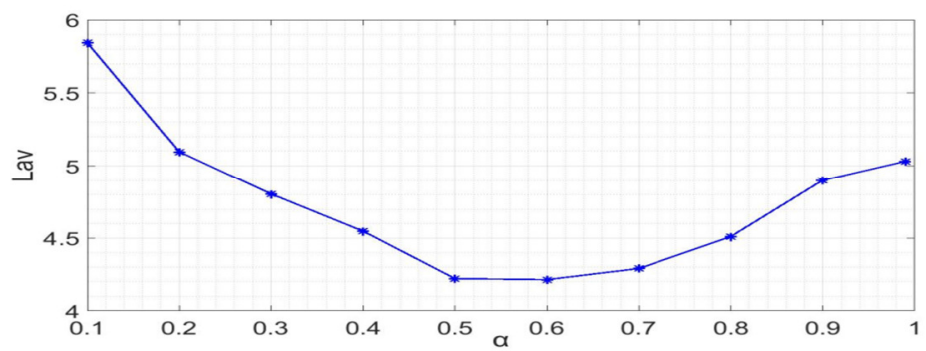

Fig. $2 L_{a v}$ vs. $\alpha$ with $L=8$ over AWGN 
Fig. 3 demonstrates the variation of BER and $L_{a v}$ against $\beta$ for the list size of 8 , with $\alpha$ set to its optimum value $\left(\alpha_{o p t}\right)$. The value of $L_{a v}$ for the proposed RP-SCL decoder is close to $L$ at $\beta$ values above 0.7 . Therefore, the considered range of $\beta$ is limited to 0.7 for all the tests. When $\beta$ is increased, the BER decays smoothly, while $L_{a v}$ increases. As a compromise between the complexity $\left(L_{a v}\right)$ and the BER performance, the value of $\beta$ at the intersection point of the two curves in Fig. 3 is considered the optimum value.

Thus, $\beta_{o p t}$ is taken to be 0.36 for $L=8$. Both $\alpha_{\text {opt }}$ and $\beta_{\text {opt }}$ are considered for determining $\gamma_{\text {opt }}$. Fig. 4 shows the decoder behavior in terms of BER and $L_{a v}$ with different values of $\gamma$ over the range of 1 to its maximum value of 1.2 (if above this value, $L_{a v}$ is close to $L=8$ ). As for the selection of $\beta_{o p t}, \gamma_{o p t}$ is the value of $\gamma$ at the intersection point of the two curves, which is 1.14 in this case.

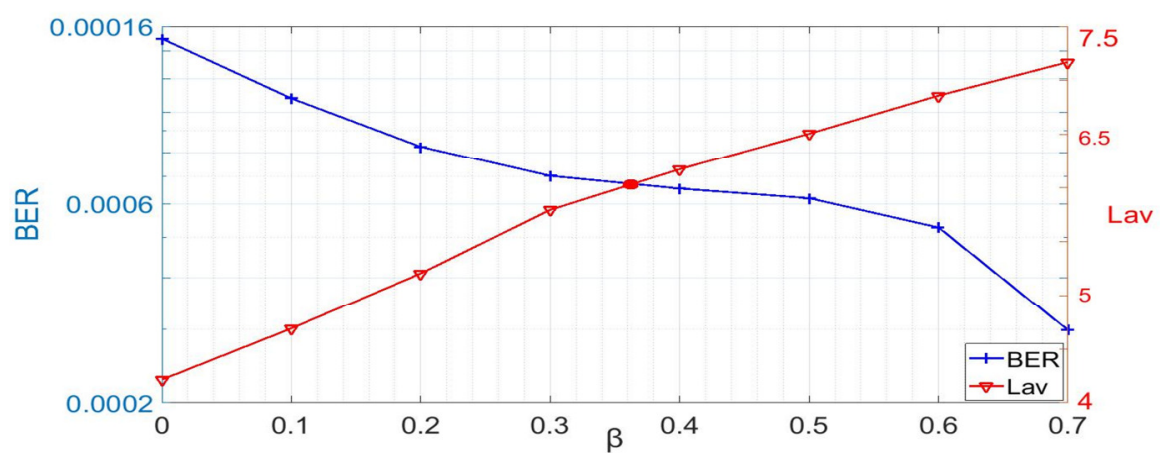

Fig. 3 BER and $L_{a v}$ vs. $\beta$ with $L=8$ over AWGN

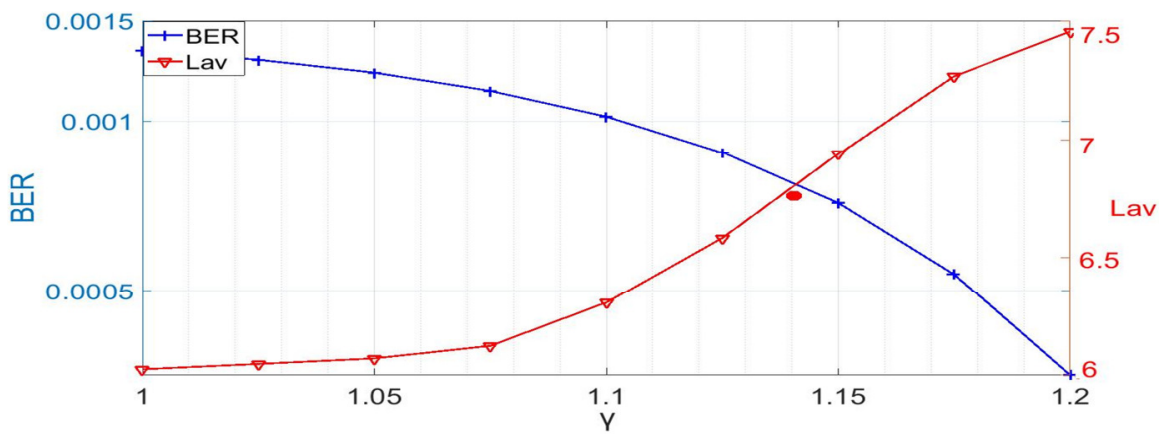

Fig. 4 BER and $L_{a v} v s . \gamma$ with $L=8$ over AWGN

The same tests as those used to determine $\alpha_{o p t}, \beta_{\text {opt }}$, and $\gamma_{o p t}$ values for AWGN using $L=8$ are performed to determine the corresponding values for the AWGN channel with $L=32$ and for the fading channel with both $L=8$ and 32 . The obtained optimum parameters are shown in Table 4.

Table 4 The RP-SCL optimum parameters for AWGN and fading channels.

\begin{tabular}{|c|c|c|c|c|}
\hline \multirow{2}{*}{ Parameter } & \multicolumn{2}{|c|}{ AWGN Channel } & \multicolumn{2}{c|}{ Fading Channel } \\
\cline { 2 - 5 } & $L=8$ & $L=32$ & $L=8$ & $L=32$ \\
\hline$\alpha_{\text {opt }}$ & 0.6 & 0.6 & 0.5 & 0.6 \\
\hline$\beta_{\text {opt }}$ & 0.36 & 0.25 & 0.379 & 0.345 \\
\hline$\gamma_{\text {opt }}$ & 1.14 & 1.1025 & 1.117 & 1.0825 \\
\hline
\end{tabular}

After the determination of the optimum value of each pruning parameter, the full operation of the proposed RP-SCL decoder proceeds as described in Section 2. The role of the RP-SCL decoder in reducing the complexity is depicted in Figs. 5-6 for the AWGN channel and the fading channel, respectively.

Each of these figures illustrates the complexity of the RP-SCL decoder in terms of the average processed paths $\left(L_{a v}\right)$ in the decoding against the SNR given by $\mathrm{E}_{\mathrm{b}} / \mathrm{N}_{\mathrm{o}}$ in decibels. Fig. 5 depicts that $L_{a v}$ is reduced by 2 and 12.5 for $L=8$ and 32 over the AWGN channel, respectively, at a high SNR. The corresponding values for the fading channel, shown in Fig. 6, are 1.8 and 10 for $L=8$ and 32 , respectively. 


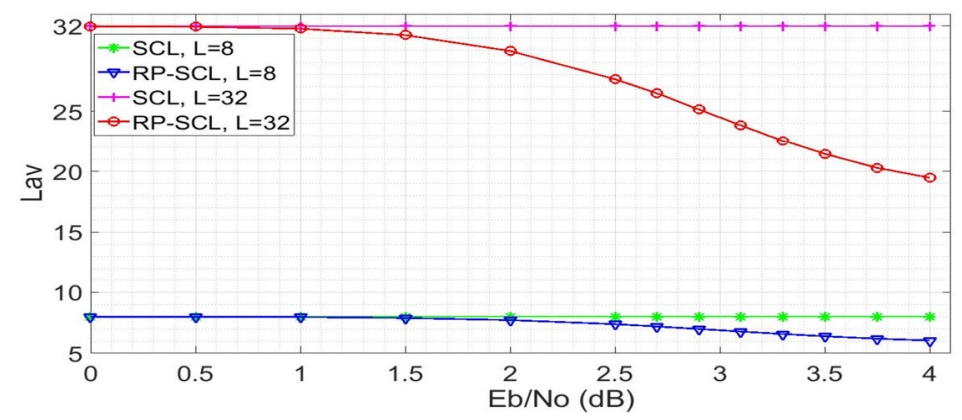

Fig. 5 The variation of $L_{a v}$ with $\mathrm{E}_{\mathrm{b}} / \mathrm{N}_{0}$ over AWGN channel

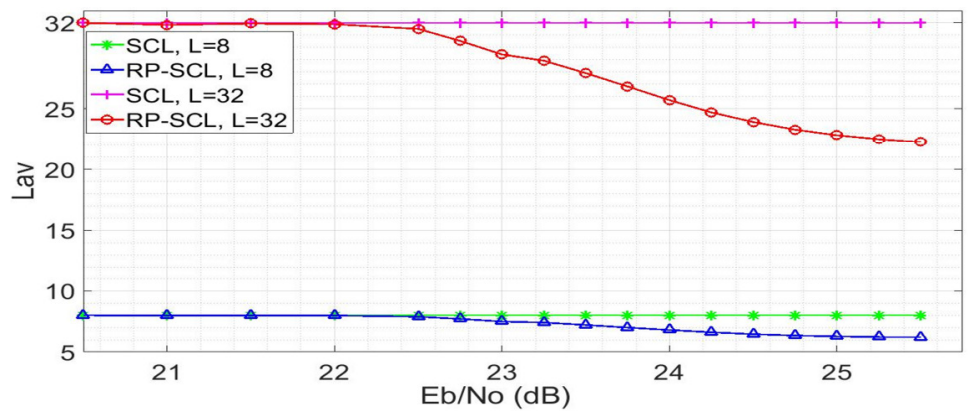

Fig. 6 The variation of $L_{a v}$ with $\mathrm{E}_{\mathrm{b}} / \mathrm{N}_{0}$ over fading channel

The results, which are presented in Table 4, demonstrates that $\alpha_{o p t}$ is less sensitive to the changed list size and channel than the other two pruning parameters $\left(\beta_{o p t}\right.$ and $\left.\gamma_{o p t}\right)$. In addition, the results reveal that $\alpha_{o p t}$ is the most important parameter for achieving the decoder complexity reduction, as shown in Fig. 2, where for $L=8$ over the AWGN channel, $L_{a v}$ is reduced to 4.2 paths at the cost of increasing the BER to $10^{-3}$. In contrast, the main role of the other pruning parameters $\beta_{\text {opt }}$ and $\gamma_{\text {opt }}$ is to mitigate the effect of $\alpha_{\text {opt }}$ on the performance, where the BER is reduced to the $10^{-4}$ range, as demonstrated in Fig. 7 with the increase of $L_{a v}, 6$, for $L=8$ over the AWGN channel.

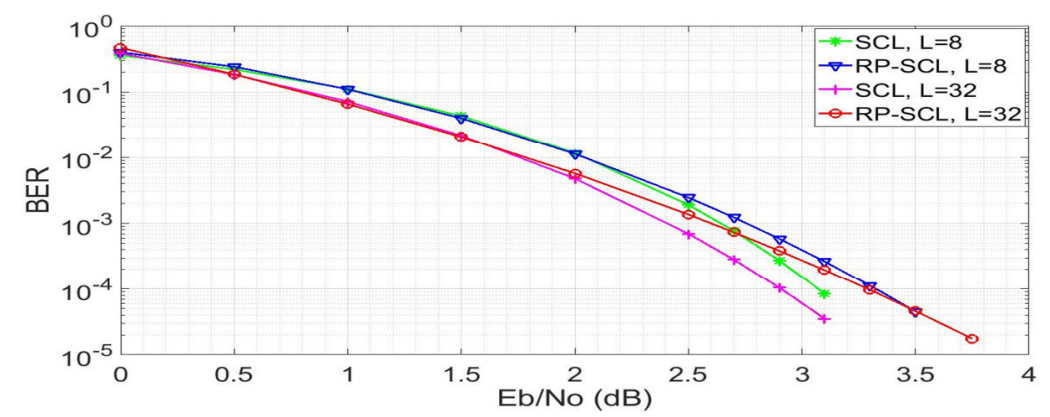

Fig. 7 The BER performance of different decoders over AWGN channel

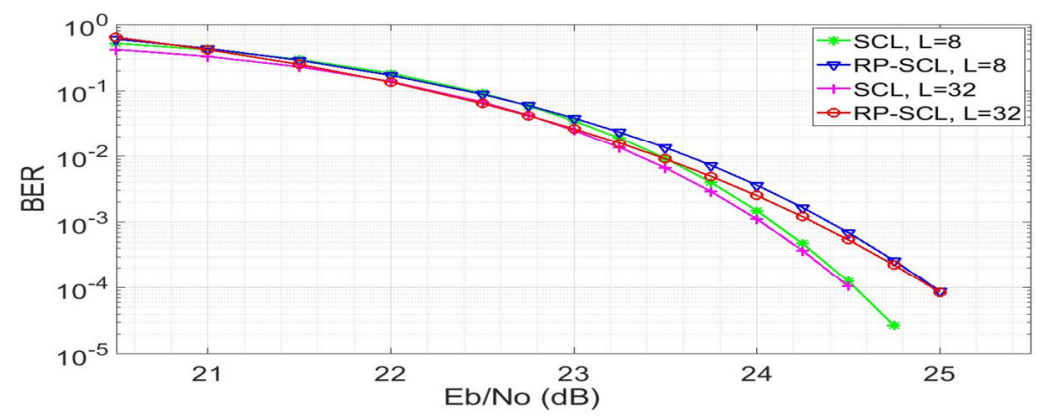

Fig. 8 The BER performance of different decoders over fading channel

Figs. 7 and 8 show the BER performance of the SCL and the RP-SCL decoders over the AWGN channel and the fading channel, respectively. The BER performance of RP-SCL is close to that of SCL at a low SNR, while relatively small degradations occurrs at a high SNR for the two channels and list sizes used. This degradation for the AWGN channel corresponds to 0.3 and $0.4 \mathrm{~dB}$ for the list sizes of 8 and 32, respectively, at the BER of $10^{-4}$, as depicted in Fig. 7 . 
The corresponding degradation for the fading channel is approximately 0.35 and $0.4 \mathrm{~dB}$ for the list sizes of 8 and 32 , respectively, as shown in Fig. 8. Such degradation can be considered to be the cost paid for the complexity reduction of RPSCL as compared to SCL.

\subsection{Complexity of the proposed RP-SCL decoder}

The performance evaluation of the proposed RP-SCL decoder together with its complexity is summarized in Table 5 for all the considered channels and list sizes. As the complexity of the SCL and RP-SCL is mainly determined by the number of processed paths, the overall reduction in $L_{a v}$ for the RP-SCL decoder as compared to that in $L$ for the SCL decoder determines the percentage of computational complexity reduction $(C C R \%)$ of the RP-SCL decoder. The value of $C C R \%$ can be calculated as:

$$
C C R \%=\frac{L-L_{a v}}{L} * 100 \%
$$

where $L$ is the list size of the SCL decoder, and $L_{a v}$ is the average number of paths processed by the RP-SCL decoder.

Table 5 The summary of the performance comparison of RP-SCL and SCL decoders

\begin{tabular}{|c|c|c|c|}
\hline & Compared term relative to that of standard SCL decoder & $L=8$ & $L=32$ \\
\hline \multirow{2}{*}{ AWGN Channel } & Percentage reduction in $\mathrm{L}_{\mathrm{av}}(\mathrm{RCC} \%)$ & $25 \%$ & $40 \%$ \\
\cline { 2 - 4 } & SNR degradation in $\mathrm{dB}$ at BER $=10^{-4}$ & 0.3 & 0.4 \\
\hline \multirow{2}{*}{ Fading Channel } & Percentage reduction in $\mathrm{L}_{\mathrm{av}}(\mathrm{RCC} \%)$ & $22.5 \%$ & $30.5 \%$ \\
\cline { 2 - 4 } & SNR degradation in $\mathrm{dB}$ at BER $=10^{-4}$ & 0.35 & 0.4 \\
\hline
\end{tabular}

Clearly, Table 5 depicts the considerable complexity reduction of the proposed RP-SCL over SCL for both the channels and the list sizes. This reduction for the list size of 8 ranges from $22.5 \%$ for the fading channel to $25 \%$ for the AWGN channel. The corresponding reduction for the list size of 32 is considerably larger. The corresponding degradation in the SNR requires to achieve a BER of $10^{-4}$ ranged from 0.3 to $0.4 \mathrm{~dB}$ over all the considered channels and list sizes.

The computational complexity for a general polar code $\mathrm{PC}(N, K)$ with an SCL decoder having a list size of $L$ is $\mathrm{O}\left(L \cdot N \cdot \log _{2} N\right)$ [2]. As the proposed decoder reduces the required $\mathrm{L}$ to $L_{a v}$ on average, one might conclude that the computational complexity of the proposed RP-SCL decoder is roughly $\mathrm{O}\left(L_{a v} \cdot N \cdot \log _{2} N\right)$.

Algorithm 1 shows that the additional mathematical operations required by the RP-SCL decoder are as follows: four multiplications, $L$ additions, and $(2 L+3)$ comparisons in the worst case for each decoding level. The required operations to determine the optimum parameters $\left(\alpha_{o p t}, \beta_{\text {opt }}\right.$, and $\gamma_{\text {opt }}$ ) of the proposed RP-SCL decoder can be carried out prior to the actual transmission and do not affect the decoding operations.

It has been shown that the expression used to estimate the total memory required for SCL decoder is as follows [12]:

$$
M_{S C L}=[N+L(N-1)] Q_{L L R}+L Q_{P M}+L(2 N-1)
$$

where $M_{S C L}$ is the total memory size (in bits) for the SCL decoder, $Q_{L L R}$ is the number of bits used to quantize LLR, and $Q_{P M}$ is the number of bits required to quantize the path metrics PM. For the proposed RP-SCL decoder, one may use the same expression with $L$ replaced by $L_{a v}$ to calculate the required memory of the RP-SCL decoder as follows:

$$
M_{R P-S C L}=\left[N+L_{a v}(N-1)\right] Q_{L L R}+L_{a v} Q_{P M}+L_{a v}(2 N-1)
$$

where $M_{R P-S C L}$ is the total required memory for the proposed RP-SCL decoder. 


\section{Conclusions}

A low-complexity decoder for polar codes was proposed in this paper. The proposed decoding algorithm, which is called reduced path successive cancellation list (RP-SCL), relies on the pruning of the decoding paths. Three parameters were used to control the pruning thresholds, which were used to perform the path pruning resulting in a considerable reduction in the decoder complexity with a slight degradation in the BER performance. These parameters were optimized to achieve the best results in terms of the complexity reduction and the least BER performance loss. The optimization was performed over the models of AWGN and mmWave fading channels. The parameters were optimized prior to the transmission so that no extra processing was required during the actual transmission. The simulation results of RP-SCL showed that a relatively high complexity reduction was achieved at a high SNR. A reduction in the complexity of up to $40 \%$ in comparison with that of the conventional SCL decoder was obtained. A similar order achieved in the required memory and computational complexity of the decoder. Such advantages were achieved on the expense of less than $0.4 \mathrm{~dB}$ SNR penalty. The implementation of RP-SCL represents a challenge to assess its suitability in practice.

\section{Conflicts of Interest}

The authors declare no conflict of interest.

\section{References}

[1] E. Arikan, "Channel Polarization: A Method for Constructing Capacity-Achieving Codes for Symmetric Binary-Input Memoryless Channels," IEEE Transaction on Information Theory, vol. 55, no. 7, pp. 3051-3073, July 2009.

[2] H. Vangala, E. Viterbo, and Y. Hong, "A Comparative Study of Polar Code Constructions for the AWGN Channel," http://arxiv.org/abs/1501.02473, January 11, 2015. (under revision)

[3] P. Giard, C. Thibeault, and W. J. Gross, High-Speed Decoders for Polar Codes, Springer International Publishing, 2017.

[4] P. Marsch, Ö. Bulakç, O. Queseth, and M. Boldi, 5G System Design Architectural and Functional Considerations and Long Term Research, John Wiley \& Sons Ltd, 2018.

[5] D. Chandramouli, R. Liebhar, and J. Pirskanen, 5G for the Connected World, John Wiley \& Sons Ltd, 2019.

[6] I. Tal and A. Vardy, "List Decoding of Polar Codes," IEEE Transactions on Information Theory, vol. 61, no. 5, pp. 2213-2226, May 2015.

[7] W. Abdulwahab and A. Kadhim, "Comparative Study of Channel Coding Schemes for 5G," Proceedings 2018 International Conference on Advanced Science and Engineering, IEEE Press, October 2018, pp. 239-243.

[8] V. Bioglio, C. Condo, and I. Land, “Design of Polar Codes in 5G New Radio," IEEE Communications Surveys and Tutorials (Early Access), January 2020.

[9] K. Chen, K. Niu, and J. Lin, "A Reduced-Complexity Successive Cancellation List Decoding of Polar Codes," Proceedings 2013 IEEE 77th Vehicular Technology Conference (VTC Spring), IEEE Press, June 2013, pp. 1-5.

[10] K. Chen, B. Li, H. Shen, J. Jin, and D. Tse, "Reduce The Complexity of List Decoding of Polar Codes by Tree-Pruning," IEEE Communication Letter, vol. 20, no. 2, pp. 204-207, February 2016.

[11] S. Hashemi, A. Balatsoukas-Stimming, P. Giara, C. Thibeaul, and W. Gross W, "Partitioned Successive-Cancellation List Decoding of Polar Codes," Proceedings of 2016 IEEE International Conference on Acoustics, Speech and Signal Processing, IEEE Press, Shanghai, China, March 2016, pp. 957-960.

[12] S. Hashemi, M. Mondelli, S. Hassani, R. Urbanke, and W. Gross, "Partitioned List Decoding of Polar Codes: Analysis and Improvement of Finite Length Performance," Proceedings of IEEE Global Communications Conference, IEEE Press, December 2017, pp. 1-7.

[13] S. Hashemi, M. Mondelli, S. Hassani, C. Condo, R. Urbanke, and W. Gross, "Decoder Partitioning: Towards Practical List Decoding of Polar Codes," IEEE Transaction on Communication, vol. 66, no. 9, pp. 3749-3759, September 2018.

[14] S. Hashemi, C. Condo, and W. Gross, "Simplified Successive-Cancellation List Decoding of Polar Codes," Proceedings of 2016 IEEE International Symposium on Information Theory, IEEE Press, July 2016, pp. 815-819.

[15] S. Hashemi, C. Condo, and W. Gross, "Fast Simplified Successive-Cancellation List Decoding of Polar Codes," Proceedings 2017 IEEE Wireless Communications and Networking Conference Workshops, IEEE Press, March 2017, pp. 1-6. 
[16] C. Condo, V. Bioglio, and I. Land, “Generalized Fast Decoding of Polar Codes,” Proceedings of 2018 IEEE Global Communications Conference, IEEE Press, December 2018, pp. 1-6.

[17] A. Balatsoukas-Stimming, M. Parizi, and A. Burg, "LLR-Based Successive Cancellation List Decoding of Polar Codes," IEEE Transaction on Signal Processing, vol. 63, no. 19, pp. 5165-5179, October 2015.

[18] Z. Gao, L. Dai, D. Mi, Z. Wang, M. A. Imran, and M. Z. Shakir, "MmWave Massive MIMO Based Wireless Backhaul for 5G Ultra-Dense Network," IEEE Wireless Communication, vol. 22, no. 5, pp. 13-21, October 2015.

[19] C. Yen and C. Liu, "Hybrid OFDM/OOK Modulations in OCDMA Scheme for Free Space Optics," Proceedings of Engineering and Technology Innovation, vol. 3, pp. 01-03, August 2016.

[20] 3rd Generation Partnership Project, "5G; NR; Physical Channels and Modulation,” TS 38.211 version 15.8.0 Release, July 15, 2018.

[21] A. Zaidi, R. Baldemair, V. Moles-Cases, N. He, K. Werner, and A. Cedergren, "OFDM Numerology Design for 5G New Radio to Support IoT, eMBB, and MBSFN,” IEEE Communication Standard Magazine, vol. 2, no. 2, pp. 78-83, June 2018.

[22] Vishwaraj and L. Ali, "Hybrid MIMO-OFDM System for 5G Network Using VLC-A Review,” Proceedings of 2019 IEEE International Conference on Electrical, Computer and Communication Technologies, February 2019, pp. 1-5.

[23] 3rd Generation Partnership Project, "5G; NR; User Equipment (UE) Radio Transmission and Reception; Part 2: Range 2 Standalone," TS 38.101-2 version 15.8.0 Release 15, January 2020.

[24] 3rd Generation Partnership Project, "5G; Study on Channel Model for Frequencies from 0.5 to 100 GHz," TR 38.901 version 14.1.1 Release, August 14, 2017.

[25] A. Sharma and M. Salim, "Polar Code: The Channel Code Contender for 5G Scenarios," Proceedings of 2017 International Conference on Computer, Communications and Electronics (Comptelix), IEEE Press, July 2017, pp. 676682.

Copyright $(\mathrm{C}$ by the authors. Licensee TAETI, Taiwan. This article is an open access article distributed under the terms and conditions of the Creative Commons Attribution (CC BY-NC) license (https://creativecommons.org/licenses/by-nc/4.0/). 